\title{
and mucin domain-3 as an emerging target for immunotherapy in cancer management
}

This article was published in the following Dove Press journal:

ImmunoTargets and Therapy

20 November 2013

Number of times this article has been viewed

\author{
Akihiro Yoneda \\ Masahisa Jinushi \\ Research Center for Infection- \\ associated Cancer, Institute for \\ Genetic Medicine, Hokkaido \\ University, Sapporo, Japan
}

Correspondence: Masahisa Jinushi Research Center for Infection-associated Cancer, Institute for Genetic Medicine, Hokkaido University, Kita I5,

Nishi 7, Kita-ku, Sapporo,

Hokkaido 060-0815, Japan

Tel +8I I | 7066073

Fax $+8 \mid$ | | $706607 \mid$

Email jinushi@igm.hokudai.ac.jp

\begin{abstract}
Cancer-induced immunosuppression significantly impacts tumors, rendering them the ability to acquire aggressive and treatment-resistant phenotypes. The recent clinical success of drugs targeting the immunosuppressive machinery of tumors highlights the importance of identifying novel drugs that effectively augment antitumor immunity and elicit clinical remission in advanced tumors. T cell immunoglobulin domain and mucin domain-3 (TIM-3) is a critical immunoregulatory molecule that links pattern recognition-mediated innate sensing with antigen-specific immune responses. Recent evidence has elucidated the potential utility of drugs targeting TIM-3 in inducing antitumor responses, particularly in synergy with conventional anticancer regimens. Herein, we provide a comprehensive overview, as well as future perspectives, regarding the role of TIM-3 as an emerging target that may improve clinical responses for cancer patients.
\end{abstract}

Keywords: tumor immunoevasion, antibody, immunosuppression, antitumor response, TIM-3

\section{Introduction}

\section{Cancer immunoevasion}

Emerging evidence has unveiled the indispensable role of the host immune system in defending itself from arising tumors. The host immune system acts to detect and eliminate tumors in three ways: 1) immune cells protect the host against viral infection and suppress virus-induced tumors, 2) innate immune cells eliminate cancer cells through activation of pattern recognition-mediated sensing systems, and 3) antigenspecific $\mathrm{T}$ cells recognize tumor rejection antigens on cancer cells. These immune system processes, which suppress tumor initiation and progression, are collectively termed "immunosurveillance."

However, rare cancer cells escape immunosurveillance and emerge as progressively growing tumors. This ability to escape recognition by the host immune system arises via various mechanisms. For example, genetic and epigenetic alterations can render tumor cells capable of reducing immune recognition (for example, by a loss of antigens). Alternatively, cancer cells may create immunosuppressive states by producing immunoregulatory cytokines such as vesicular endothelial growth factor (VEGF), transforming growth factor-beta, galectin-1, indoleamine 2,3-dioxygenase or by recruiting regulatory immune cells (forkhead box P3 [Foxp3] ${ }^{+}$regulatory T cells and myeloid-derived suppressor cells) that function as effectors of immunosuppression. ${ }^{1-8}$ In addition, host immune systems can also promote tumor progression by the selection of cancer cells that thrive, thus supporting tumor progression and anticancer drug 
resistance in privileged tumor microenvironments (TMEs). As a result, cancer cells are able to manipulate host immune systems to further enhance tumorigenicity. ${ }^{9,10}$

Cancer-induced immunoevasion is a major player in the suppression of the antitumor efficacy of immunotherapy. ${ }^{11,12}$ Moreover, cancer-mediated immunomodulation has a profound impact on many antitumor therapies including chemotherapy and molecular targeting strategies. ${ }^{13,14}$ Therefore, a deeper understanding of the molecular machineries by which cancer-induced immunomodulation influences the therapeutic responses to anticancer regimens is necessary in order to devise new strategies to improve the clinical prognosis of cancer patients.

Recent evidence has revealed that $\mathrm{T}$ cell immunoglobulin (Ig) domain and mucin domain (TIM)-3 functions as a critical checkpoint, regulating numerous aspects of tumor immunomodulation. ${ }^{15}$ This review provides an overview of the immunoregulatory functions of TIM-3 and perspectives regarding the potential of a TIM-3-targeted strategy as a new option in treating cancer patients.

\section{TIM-3: brief overview of physiological functions}

TIM-3 was identified as a molecule expressed on interferon (IFN) $\gamma$-producing CD4+ T-helper type 1 (Th1) and cluster of differentiation (CD)8+ T-cytotoxic type 1 (Tc1) cells. ${ }^{15}$ TIM-3 belongs to the TIM family of molecules that, in mice, contains eight members. Only TIM-1, TIM-3, and TIM-4 are expressed in humans. TIM-3 consists of an N-terminal $\mathrm{IgV}$ domain followed by a mucin domain, a transmembrane domain, and a cytoplasmic tail (Figure 1). Four noncanonical cysteines in the IgV domain are conserved in all TIM-family genes across mice and humans, forming a unique binding cleft not seen in the Ig domain of any other Ig superfamily
Mouse

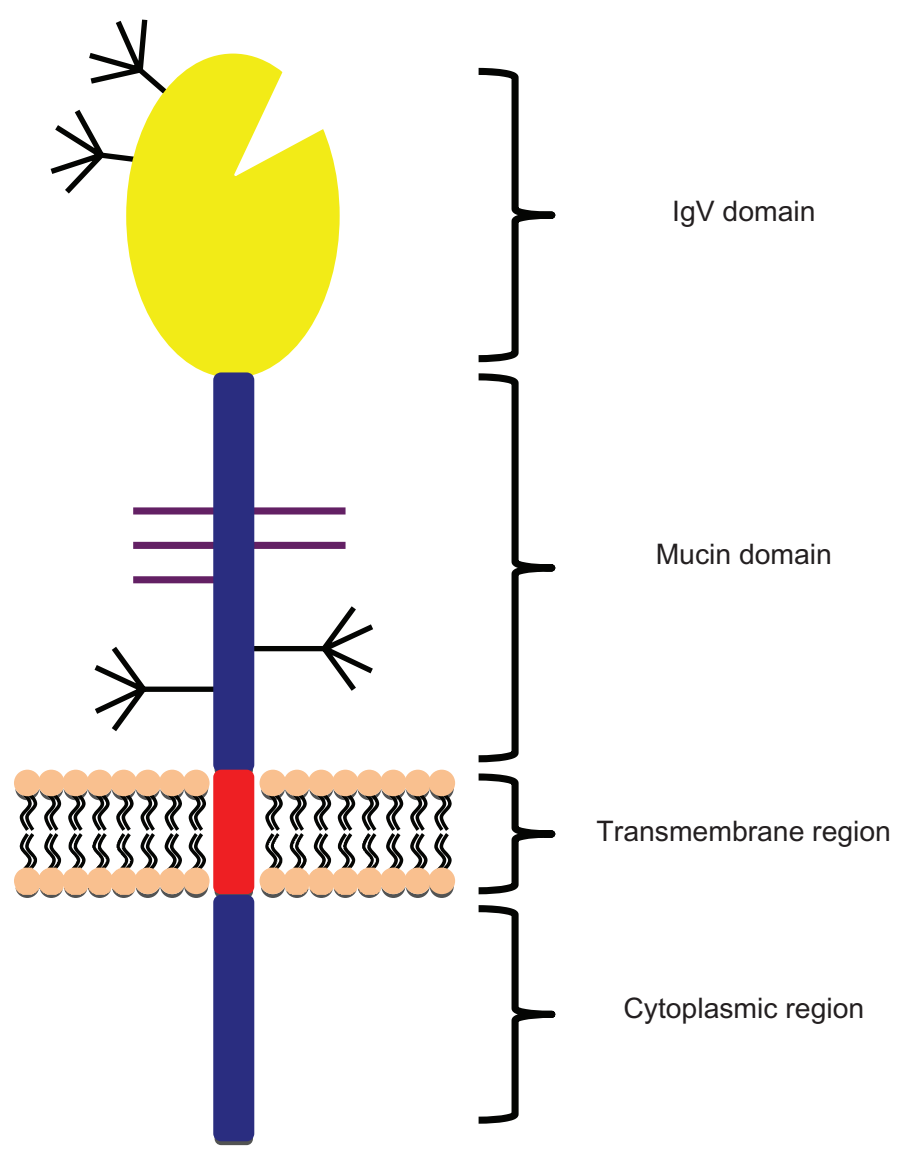

Human

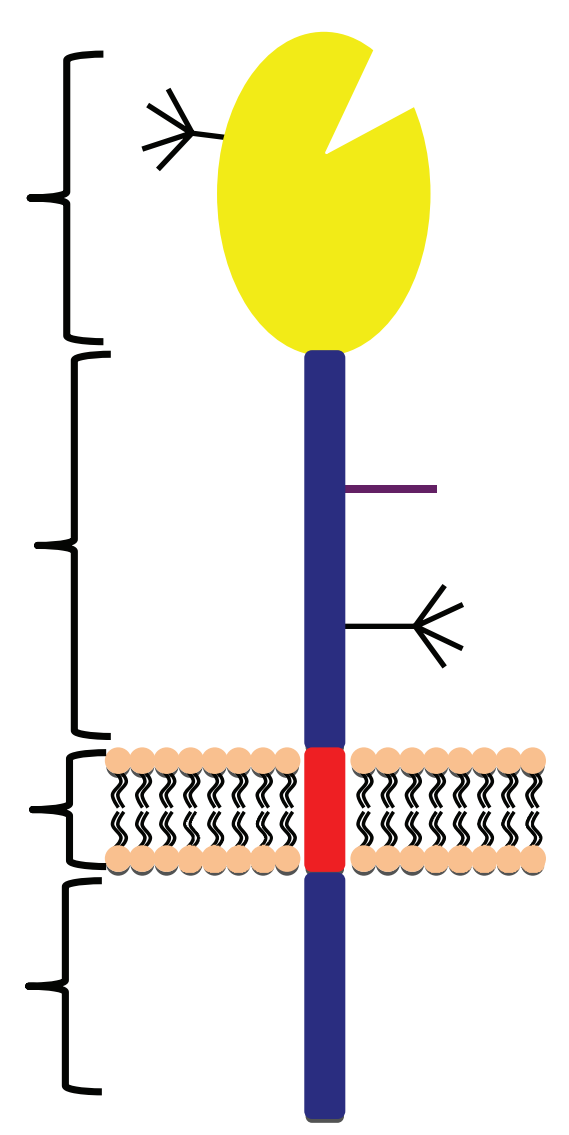

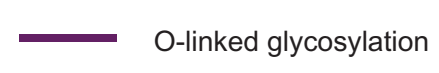

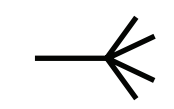

N-linked glycosylation

Figure I Schema of human and mouse T cell immunoglobulin domain and mucin domain-3 (TIM-3) protein structures. TIM-3 consists of an N-terminal immunoglobulin (Ig) $\vee$ domain followed by a mucin domain, transmembrane region, and a cytoplasmic region. The $\lg \mathrm{V}$ and mucin domains contain $\mathrm{O}$ - and $\mathrm{N}$-linked glycosylation sites. 
member. ${ }^{16,17}$ Interestingly, the cleft structure surrounded by an $\alpha$-loop in TIM-3 has been shown to be important for binding phosphatidylserine (PS), which mediates uptake of apoptotic cells by macrophages and dendritic cells. ${ }^{18,19}$

TIM-3 interacts with multiple ligands, including galectin-9 (Ga19) and cell-surface PS, leading to various biological consequences. The binding of TIM-3 to Gal9 or high mobility group protein B1 (HMGB1) generates an inhibitory signal that results in the apoptosis of Th1 cells. ${ }^{20,21}$ Continuous exposure to interleukin 12 induces TIM-3 expression on intra-tumor $\mathrm{T}$ cells, triggering functional impairment and exhaustion. ${ }^{22}$ TIM-3 expressed on other immune cells such as natural killer cells and dendritic cells (DCs) is also involved in immunoregulatory functions. For example, TIM-3 regulates the differentiation and immunogenic activities of natural killer cells. ${ }^{23,24}$ TIM-3 expressed on DCs promotes the phagocytosis of apoptotic cells through interaction with PS, which enhances antigen presentation and triggers immune tolerance, ${ }^{18,19}$ whereas it negatively regulates pattern recognition-mediated innate immune systems. ${ }^{25}$ Further, TIM-3 synergizes with Toll-like receptors to induce inflammation by activating the transcription factor nuclear factor kappa B and enhancing the secretion of pro-inflammatory mediators. $^{26}$

Together, these findings suggest that TIM-3 controls both the tolerogenic and immunostimulatory properties of various subsets of immune cells. These dual TIM-3 functions may be regulated differentially at various stages of tumorigenicity and/or by distinct TME subtypes (Figure 2).

\section{Role of TIM-3 in cancer immunosuppression}

Accumulating evidence has revealed that TIM-3 plays a critical role in negatively regulating $\mathrm{T}$ cell-dependent

A

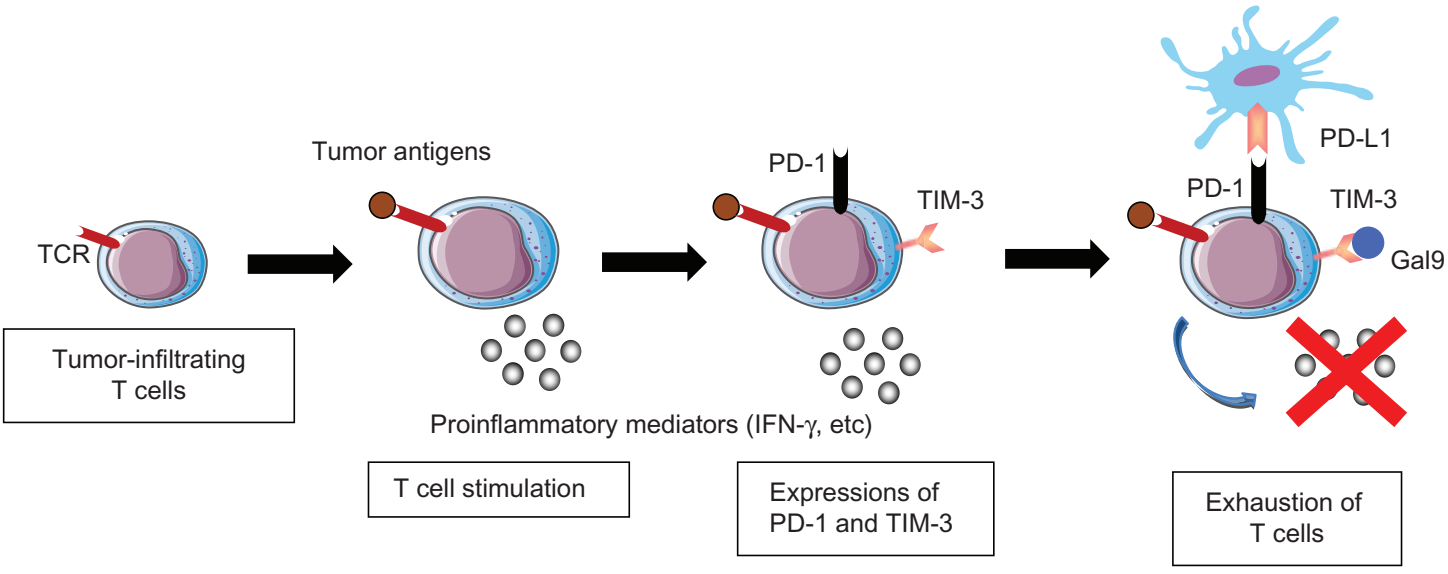

B

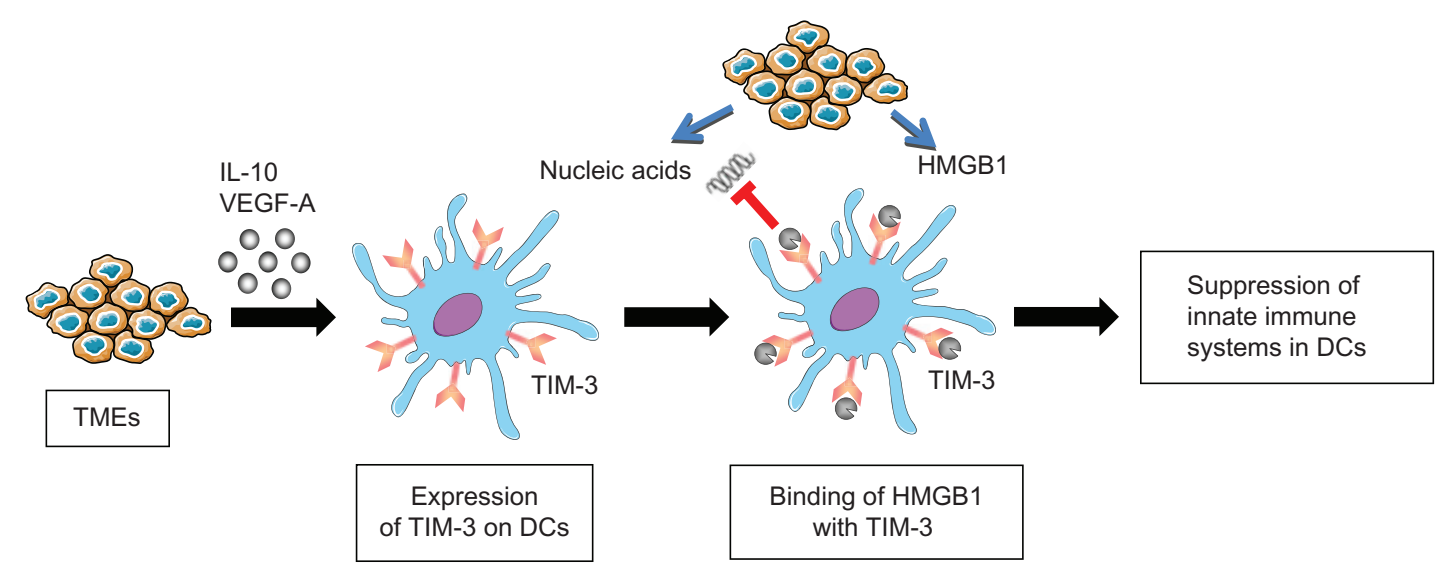

Figure 2 Role of T cell immunoglobulin domain and mucin domain-3 (TIM-3) in cancer immunosuppression.

Notes: (A) Exhaustion of T cells by TIM-3 and programmed cell death protein I (PD-I). Tumor-infiltrating T cells secrete interferon- $\gamma$ (IFN $\gamma$ ) upon binding of tumor antigens by $T$ cell receptors (TCR). However, TIM-3 and PD-I are upregulated on tumor-infiltrating T cells upon chronic exposure to antigenic stimuli, and interact with galectin- 9 (Gal9) and programmed cell death I ligand I (PD-LI) expressed on tumors or tumor-infiltrating stromal cells. These interactions impair the effector activities of tumorinfiltrating T cells, leading to cancer immunosuppression. (B) Immunosuppression of dendritic cells (DCs) by TIM-3. TIM-3 expression on DCs is induced via stimulation by interleukin (IL) 10 and vascular endothelial growth factor A (VEGF-A), which are mainly secreted from tumor microenvironments (TMEs). TIM-3 on DCs binds high mobility group box I (HMGBI) from inflammatory TMEs, and negatively regulates the HMGBI-mediated recruitment of TMR-derived nucleic acids, thereby suppressing the innate immune systems. As a result, TIM-3 on DCs enables tumors to acquire immunosuppressive capabilities. Abbreviations: TCR, T cell receptor, VEGF, vascular endothelial growth factor; DC, dendritic cells. 
immunosurveillance. For example, the exhausted phenotype of tumor-infiltrating $\mathrm{T}$ cells is manifested by the coexpression of TIM-3 and programmed cell death protein 1 (PD-1), which is closely correlated with dampened tumor antigen-specific responses. ${ }^{27,28}$ In addition, TIM-3 expression may represent a distinct immunosuppressive feature of Foxp $3^{+}$regulatory $\mathrm{T}$ cells, although the relative importance of this population compared with other subsets requires clarification. ${ }^{29}$ These findings collectively suggest that TIM-3 functions as a critical negative regulator of tumor-specific recognition by $\mathrm{T}$ cells.

Immune regulation by innate immune cells has emerged as an indispensable defense system against tumor growth and progression. ${ }^{30}$ Consistent with the role of TIM-3 in regulating antitumor innate immune responses, together with colleagues, we recently showed that TIM-3 functions to suppress the nucleic acid-mediated innate immune responses of tumor-infiltrating DCs. ${ }^{26}$ TIM-3 is expressed on DCs in tumors at much higher levels than on DCs in normal tissues, and preferentially binds HMGB1, which has a critical role in stimulating nucleic acid-mediated innate immunity. ${ }^{31,32}$ TIM-3 negatively regulates the HMGB1-mediated recruitment of nucleic acids to the endosomal compartment of DCs, thus shutting down the downstream signaling cascades mediated by Toll-like receptors and cytosolic sensors. These results demonstrate that TIM-3 on DCs enables tumors to evade immunosurveillance by attenuating the sensing of nucleic acids that are potentially released upon anticancer treatment and/or endogenous tumor-associated inflammation. ${ }^{26}$

More importantly, recent clinical studies have revealed that TIM-3 may serve as a prognostic marker to predict poor responses to anticancer therapies and, therefore, poor survival in patients with non-small cell lung carcinomas and clear-cell renal-cell carcinomas. ${ }^{33,34}$

Together, TIM-3 serves as a negative regulator of both innate and antigen-specific immune responses, thereby establishing an ideal environment for tumor immunoevasion and tumorigenicity.

\section{Targeting immunoevasion by anticancer drugs: brief overview of current status}

Recent advances in clarifying the molecular and cellular mechanisms of immune regulation of tumors have led to breakthroughs for developing novel drugs that specifically target the immunoregulatory functions of lymphocytes and myeloid cells. ${ }^{35,36}$ In particular, the targeting of immune checkpoint molecules is currently at the forefront of cancer immunotherapy. ${ }^{37}$ Treatment with an anti-cytotoxic T-lymphocyte antigen 4 (CTLA-4) (ipilimumab) has extended the overall survival of patients with advanced melanomas compared with vaccination with gp100 peptide, and the combined regimens of anti-CTLA-4 with dacarbazine significantly improved the overall survival of previously untreated melanoma patients compared with dacarbazine alone. ${ }^{38,39}$ Moreover, early clinical studies revealed that monoclonal antibody (mAb)-mediated targeting of PD-1 (nivolumab), programmed cell death 1 ligand 1 , or the combination of PD-1 and CTLA-4 elicited potent antitumor immune responses and improved clinical responses in patients with metastatic melanomas, non-small cell lung carcinomas or renal-cell carcinomas. ${ }^{40-42}$ The activation of CD40 by an agonistic $\mathrm{mAb}$ (CP-870,893) promoted immunogenic activities of tumorinfiltrating macrophages, which led to the differentiation of tumoricidal stromata and triggered clinical responses in patients with pancreatic cancer. ${ }^{43}$ These results decisively provide a proof-of-concept that the reversal of tumor-induced immunomodulation by $\mathrm{mAb}$ changes the clinical prognosis in patients with treatment-refractory cancers. Additionally, adoptive transfer of $\mathrm{T}$ lymphocytes engineered to express chimeric antigen receptor targeting tumor-specific antigens resulted in potent antitumor immune responses and long-term remission in some patients with chronic lymphoid leukemia and solid malignancies. ${ }^{44,45}$

More importantly, the profound effects of several immune-targeting drugs have prompted researchers and pharmaceutical industries to develop novel immunotherapeutic drugs that target tumors and their immunosuppressive microenvironments. These are mainly comprised of mAbs targeting immune checkpoint and regulatory $\mathrm{T}$ cell functions, such as anti-OX40 antibody, anti-CD137 antibody, anti-glucocorticoid-induced tumor necrosis factor receptorrelated protein antibody, etc. ${ }^{36,46-48}$ Together, these advances in the clinical development of immune-targeting drugs in coordination with scientific advances in understanding tumor immune responses should have a profound impact on constructing new strategies to combat malignant diseases in clinical settings.

\section{The potential for targeting TIM-3 as a new anticancer drug}

Recent studies have identified TIM-3 as a promising candidate for a therapeutic target that may effectively trigger antitumor immune responses and clinical remission in cancer patients. In a preclinical study utilizing murine tumor models, treatment with an anti-TIM-3 mAb (RMT3-23) resulted in 
substantial tumor regression by stimulating tumor-specific T cell immunity. ${ }^{49}$ Further, the anti-TIM-3 mAb synergized with anti-PD-1 mAb to augment antitumor responses by eliminating exhausted T cells in tumor tissues..$^{25,26,50}$ Therefore, the pharmacological manipulation of TIM-3 functions results in the reversion of immunosuppressive TMEs and thus leads to profound improvements in anticancer responses in coordination with other treatment regimens including immunotherapy, chemotherapy, radiotherapy, and certain subsets of molecular targeting.

In accordance with our assumptions about the role of TIM-3 in the regulation of anticancer drug responses, with colleagues, we demonstrated that treatment with murine or human anti-TIM-3 mAb (RMT3-23 and ATIK2a, respectively) augmented antitumor responses to cytotoxic chemotherapy or immunotherapy by increasing the antitumor immunogenicity of nucleic acids released from TMEs. ${ }^{25,51}$ Anticancer chemotherapies trigger the release of endogenous inflammatory mediators termed "damage-associated molecular pattern" (DAMP) molecules, such as HMGB1, adenosine triphosphate, and DNA, which serve as enriched sources for innate immune signals. ${ }^{52}$ Thus, the drugs targeting TIM-3mediated anti-inflammatory responses enhance the antitumor effects of chemotherapy by sensitizing DAMP moleculedependent innate immune surveillance. Moreover, recent evidence has revealed the importance of innate immune sensing systems in effectively triggering antigen-specific effector responses and differentiating memory T cells. ${ }^{53}$ Thus, it is tempting to speculate that TIM-3 stimulates antitumor innate sensing and immunological memory in synergy with inflammatory responses evoked by conventional anticancer regimens, thus opening new perspectives in the field of chemo-immunotherapy.

Despite these promising findings, which may pave the way for developing new and innovative immune targets and therapies for cancer patients, several issues remain unresolved regarding the role of TIM-3 in the regulation of antitumor responses to tumor immunotherapy in the course of tumor immunosurveillance and tumorigenic inflammation. Since TIM-3 serves as a negative regulator of both the innate and adaptive arms of immunity, blockade of TIM-3 should improve the therapeutic efficacy of immunotherapies in the early phase of tumor immunosurveillance. In contrast, the inhibition of TIM-3 may be detrimental in augmenting antitumor responses induced by immunotherapy in the background of pro-tumorigenic inflammation. Thus, our hypothesis highlights the complex and dual features of TIM-3 in the regulation of therapeutic responses to anticancer modalities including immunotherapy.

In addition, the expression and function of TIM-3 in TME components other than immune cells, such as endothelial cells, fibroblasts, and so on, remains largely, un explored. Recent studies have identified TIM-3 as a functional marker that is specifically expressed on acute myeloid leukemia stem cells, raising the possibility that TIM-3 controls tumorigenicity by creating complex networks formed by tumorigenic cells and tumor-associated non-transformed cells. ${ }^{54-56}$ Further studies of the origins, inducers, and functional specialization of TIM-3-expressing cells in the tumor environment will help to clarify how TMEs regulate the balance between antitumor immunosurveillance and tumor-promoting inflammation.

It also remains unclear how ligation of TIM-3 on different cell types can mediate the multiple immunoregulatory effects of different immune cell subsets. One key feature of TIM-3-mediated immune regulation is that TIM-3 has many ligands through which to exert multiple aspects of its biological activities. For example, Gal9 interacts with TIM-3 through amino-linked carbohydrates, whereas HMGB1 and PS bind to TIM-3 through a metal ion-dependent ligandbinding site in the FG loop of the Ig variable domain. ${ }^{17,18,26}$ The binding of each ligand to the extracellular site of its receptor leads to conformational changes in the cytoplasmic tail, which activate alternative signaling pathways and thus exert distinct biological actions. Alternatively, it is tempting to speculate that post-translational modifications such as glycosylation may have a great impact on altering the immunological functions of TIM-3 in TMEs. Further studies should elucidate whether TIM-3 regulates pro- and antiinflammatory responses by selecting distinct ligands and/or that such regulation is mediated through post-transcriptional modification of TIM-3 itself.

\section{Conclusion}

Tumor immunosuppression has emerged as an important hallmark of tumorigenicity. ${ }^{57}$ Therapeutic intervention to correct immunosuppressive micro-environments may greatly improve the antitumor effects of cancer treatments, which include non-immunotherapeutic regimens such as chemotherapy and molecular target therapy. ${ }^{58,59}$ Given the recent impressive achievements in developing novel cancer immunotherapies, the therapeutic strategies of combining conventional anticancer modalities with the modification of immunosuppressive TMEs is a profoundly promising strategy for establishing long-term regression of aggressive tumors. Importantly, TIM-3 has been specifically detected on tumorigenic acute 
myeloid leukemia cells and tumor-associated myeloid cells in contrast to non-tumor counterparts, justifying the targeting of TIM-3 as a new tumor-specific antitumor strategy. ${ }^{26,53,56}$ Thus, further understanding of the mechanisms for cancer-induced immunoevasion and the additional development of methods to overcome this evasion are critical to improve clinical remission in cancer patients in the future.

\section{Acknowledgment}

This study was partially supported by a Grant-in-Aid for Scientific Research and Scientific Research for Innovative Areas from the Ministry of Education, Culture, Sports, Science and Technology and the Ministry of Health, Labor and Welfare (MEXT), Japan.

\section{Disclosure}

The authors declare no conflicts of interest in this work.

\section{References}

1. Vesely MD, Kershaw MH, Schreiber RD, Smyth MJ. Natural innate and adaptive immunity to cancer. Annu Rev Immunol. 2011;29:235-271.

2. Dunn GP, Old LJ, Schreiber RD. The three Es of cancer immunoediting. Annu Rev Immunol. 2004;22:329-360.

3. Smyth MJ, Dunn GP, Schreiber RD. Cancer immunosurveillance and immunoediting: the roles of immunity in suppressing tumor development and shaping tumor immunogenicity. Adv Immunol. 2006;90:1-50.

4. Khong HT, Restifo NP. Natural selection of tumor variants in the generation of "tumor escape" phenotypes. Nat Immunol. 2002;3(11): 999-1005.

5. Flavell RA, Sanjabi S, Wrzesinski SH, Licona-Limón P. The polarization of immune cells in the tumour environment by TGFbeta. Nat Rev Immunol. 2010;10(8):554-567.

6. Katz JB, Muller AJ, Prendergast GC. Indoleamine 2,3-dioxygenase in T-cell tolerance and tumoral immune escape. Immunol Rev. 2008;222:206-221.

7. Rabinovich GA, Croci DO. Regulatory circuits mediated by lectin-glycan interactions in autoimmunity and cancer. Immunity. 2012;36(3):322-335.

8. Nishikawa H, Sakaguchi S. Regulatory T cells in tumor immunity. Int J Cancer. 2010;127(4):759-767.

9. Kuraishy A, Karin M, Grivennikov SI. Tumor promotion via injury- and death-induced inflammation. Immunity. 2011;35(4):467-477.

10. DeNardo DG, Johansson M, Coussens LM. Immune cells as mediators of solid tumor metastasis. Cancer Metastasis Rev. 2008;27(1):11-18.

11. Drake CG, Jaffee E, Pardoll DM. Mechanisms of immune evasion by tumors. Adv Immunol. 2006;90:51-81.

12. Rosenberg SA, Yang JC, Restifo NP. Cancer immunotherapy: moving beyond current vaccines. Nat Med. 2004;10(9):909-915.

13. Galluzzi L, Senovilla L, Zitvogel L, Kroemer G. The secret ally: immunostimulation by anticancer drugs. Nat Rev Drug Discov. 2012;11(3): 215-233.

14. Lake RA, Robinson BW. Immunotherapy and chemotherapy a practical partnership. Nat Rev Cancer. 2005;5(5):397-405.

15. Monney L, Sabatos CA, Gaglia JL, et al. Th1-specific cell surface protein Tim-3 regulates macrophage activation and severity of an autoimmune disease. Nature. 2002;415(6871):536-541.

16. Santiago C, Ballesteros A, Tami C, Martínez-Muñoz L, Kaplan GG, Casasnovas JM. Structures of T Cell immunoglobulin mucin receptors 1 and 2 reveal mechanisms for regulation of immune responses by the TIM receptor family. Immunity. 2007;26(3):299-310.
17. Cao E, Zang X, Ramagopal UA, et al. T cell immunoglobulin mucin-3 crystal structure reveals a galectin-9-independent ligand-binding surface. Immunity. 2007;26(3):311-321.

18. Nakayama M, Akiba H, Takeda K, et al. Tim-3 mediates phagocytosis of apoptotic cells and cross-presentation. Blood. 2009;113(16): 3821-3830.

19. DeKruyff RH, Bu X, Ballesteros A, et al. T cell/transmembrane, Ig, and mucin-3 allelic variants differentially recognize phosphatidylserine and mediate phagocytosis of apoptotic cells. J Immunol. 2010;184(4): 1918-1930.

20. Zhu C, Anderson AC, Schubart A, et al. The Tim-3 ligand galectin-9 negatively regulates $\mathrm{T}$ helper type 1 immunity. Nat Immunol. 2005; 6(12):1245-1252.

21. Rangachari M, Zhu C, Sakuishi K, et al. Bat3 promotes T cell responses and autoimmunity by repressing Tim-3-mediated cell death and exhaustion. Nat Med. 2012;18(9):1394-1400.

22. Yang ZZ, Grote DM, Ziesmer SC, et al. IL-12 upregulates TIM-3 expression and induces $\mathrm{T}$ cell exhaustion in patients with follicular B cell non-Hodgkin lymphoma. J Clin Invest. 2012;122(4):1271-1282.

23. Ndhlovu LC, Lopez-Vergès S, Barbour JD, et al. Tim-3 marks human natural killer cell maturation and suppresses cell-mediated cytotoxicity. Blood. 2012;119(16):3734-3743.

24. Gleason MK, Lenvik TR, McCullar V, et al. Tim-3 is an inducible human natural killer cell receptor that enhances interferon gamma production in response to galectin-9. Blood. 2012;119(13): 3064-3072.

25. Anderson AC, Anderson DE, Bregoli L, et al. Promotion of tissue inflammation by the immune receptor Tim-3 expressed on innate immune cells. Science. 2007;318(5853):1141-1143.

26. Chiba S, Baghdadi M, Akiba H, et al. Tumor-infiltrating DCs suppress nucleic acid-mediated innate immune responses through interactions between the receptor TIM-3 and the alarmin HMGB1. Nat Immunol. 2012;13(9):832-842.

27. Fourcade J, Sun Z, Benallaoua M, et al. Upregulation of Tim-3 and PD-1 expression is associated with tumor antigen-specific CD8+ T cell dysfunction in melanoma patients. J Exp Med. 2010;207(10):2175-2186.

28. Sakuishi K, Apetoh L, Sullivan JM, Blazar BR, Kuchroo VK, Anderson AC. Targeting Tim-3 and PD-1 pathways to reverse T cell exhaustion and restore anti-tumor immunity. J Exp Med. 2010;207(10): 2187-2194.

29. Yan J, Zhang Y, Zhang JP, Liang J, Li L, Zheng L. Tim-3 expression defines regulatory T cells in human tumors. PLoS One. 2013;8(3): e58006.

30. Hayakawa Y, Smyth MJ. Innate immune recognition and suppression of tumors. Adv Cancer Res. 2006;95:293-322.

31. Tian J, Avalos AM, Mao SY, et al. Toll-like receptor 9-dependent activation by DNA-containing immune complexes is mediated by HMGB1 and RAGE. Nat Immunol. 2007;8(5):487-496.

32. Yanai H, Ban T, Wang Z, et al. HMGB proteins function as universal sentinels for nucleic-acid-mediated innate immune responses. Nature. 2009;462(7269):99-103.

33. Bai J, Li X, Tong D, Shi W, Song H, Li Q. T-cell immunoglobulinand mucin-domain-containing molecule 3 gene polymorphisms and prognosis of non-small-cell lung cancer. Tumour Biol. 2013;34(2): 805-809.

34. Yuan J, Jiang B, Zhao H, Huang Q. Prognostic implication of TIM-3 in clear cell renal cell carcinoma. Neoplasia. Epub September 20, 2013.

35. Mellman I, Coukos G, Dranoff G. Cancer immunotherapy comes of age. Nature. 2011;480(7378):480-489.

36. Sharma P, Wagner K, Wolchok JD, Allison JP. Novel cancer immunotherapy agents with survival benefit: recent successes and next steps. Nat Rev Cancer. 2011;11(11):805-812.

37. Pardoll DM. The blockade of immune checkpoints in cancer immunotherapy. Nat Rev Cancer. 2012;12(4):252-264.

38. Hodi FS, O'Day SJ, McDermott DF, et al. Improved survival with ipilimumab in patients with metastatic melanoma. $N$ Engl J Med. 2010;363(8):711-723. 
39. Robert C, Thomas L, Bondarenko I, et al. Ipilimumab plus dacarbazine for previously untreated metastatic melanoma. $N$ Engl J Med. 2011;364(26):2517-2526.

40. Topalian SL, Hodi FS, Brahmer JR, et al. Safety, activity, and immune correlates of anti-PD-1 antibody in cancer. N Engl J Med. 2012;366(26): 2443-2454.

41. Brahmer JR, Tykodi SS, Chow LQ, et al. Safety and activity of antiPD-L1 antibody in patients with advanced cancer. $N$ Engl J Med. 2012;366(26):2455-2465.

42. Wolchok JD, Kluger H, Callahan MK, et al. Nivolumab plus ipilimumab in advanced melanoma. $N$ Engl J Med. 2013;369(2):122-133.

43. Beatty GL, Chiorean EG, Fishman MP, et al. CD40 agonists alter tumor stroma and show efficacy against pancreatic carcinoma in mice and humans. Science. 2011;331(6024):1612-1616.

44. Porter DL, Levine BL, Kalos M, Bagg A, June CH. Chimeric antigen receptor-modified T cells in chronic lymphoid leukemia. $N$ Engl J Med. 2011;365(8):725-733.

45. Kalos M, June CH. Adoptive T cell transfer for cancer immunotherapy in the era of synthetic biology. Immunity. 2013;39(1):49-60.

46. Weinberg AD, Morris NP, Kovacsovics-Bankowski M, Urba WJ, Curti BD. Science gone translational: the OX40 agonist story. Immunol Rev. 2011;244(1):218-231.

47. Lynch DH. The promise of 4-1BB (CD137)-mediated immunomodulation and the immunotherapy of cancer. Immunol Rev. 2008;222:277-286.

48. Schaer DA, Cohen AD, Wolchok JD. Anti-GITR antibodies - potential clinical applications for tumor immunotherapy. Curr Opin Investig Drugs. 2010;11(12):1378-1386.

49. Ngiow SF, von Scheidt B, Akiba H, Yagita H, Teng MW, Smyth MJ. Anti-TIM3 antibody promotes T cell IFN- $\gamma$-mediated antitumor immunity and suppresses established tumors. Cancer Res. 2011;71(10): 3540-3551.
50. Zhou Q, Munger ME, Veenstra RG, et al. Coexpression of Tim-3 and PD-1 identifies a CD8+ T-cell exhaustion phenotype in mice with disseminated acute myelogenous leukemia. Blood. 2011;117(17): 4501-4510.

51. Baghdadi M, Nagao H, Yoshiyama H, et al. Combined blockade of TIM-3 and TIM-4 augments cancer vaccine efficacy against established melanomas. Cancer Immunol Immunother. 2013;62(4):629-637.

52. Krysko DV, Garg AD, Kaczmarek A, Krysko O, Agostinis P, Vandenabeele P. Immunogenic cell death and DAMPs in cancer therapy. Nat Rev Cancer. 2012;12(12):860-875.

53. Iwasaki A, Medzhitov R. Regulation of adaptive immunity by the innate immune system. Science. 2010;327(5963):291-295.

54. Jan M, Chao MP, Cha AC, et al. Prospective separation of normal and leukemic stem cells based on differential expression of TIM3, a human acute myeloid leukemia stem cell marker. Proc Natl Acad Sci U S A. 2011;108(12):5009-5014.

55. Kikushige Y, Shima T, Takayanagi S, et al. TIM-3 is a promising target to selectively kill acute myeloid leukemia stem cells. Cell Stem Cell. 2010;7(6):708-717.

56. Huang X, Bai X, Cao Y, et al. Lymphoma endothelium preferentially expresses Tim-3 and facilitates the progression of lymphoma by mediating immune evasion. J Exp Med. 2010;207(3):505-520.

57. Hanahan D, Weinberg RA. Hallmarks of cancer: the next generation. Cell. 2011;144(5):646-674.

58. Zitvogel L, Galluzzi L, Smyth MJ, Kroemer G. Mechanism of action of conventional and targeted anticancer therapies: reinstating immunosurveillance. Immunity. 2013;39(1):74-88.

59. Knight DA, Ngiow SF, Li M, et al. Host immunity contributes to the anti-melanoma activity of BRAF inhibitors. J Clin Invest. 2013; 123(3):1371-1381.
ImmunoTargets and Therapy

\section{Publish your work in this journal}

ImmunoTargets and Therapy is an international, peer-reviewed open access journal focusing on the immunological basis of diseases, potential targets for immune based therapy and treatment protocols employed to improve patient management Basic immunology and physiology of the immune system in health, and disease will be also covered. In addition, the journal will focus on the impact of manage-

\section{Dovepress}

ment programs and new therapeutic agents and protocols on patient perspectives such as quality of life, adherence and satisfaction. The manuscript management system is completely online and includes a very quick and fair peer-review system, which is all easy to use. Visit http://www.dovepress.com/testimonials.php to read real quotes from published authors. 